\title{
Influence of Arc Flash Performance and ESDD Measurement of Bushings Tainted by Nitrates
}

\author{
R.Nikkitha, L.Kalaivani
}

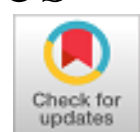

\begin{abstract}
Tainting devastate the feat of bushings. Conductors are insulated inside the bushing that carry a high voltage current through a grounded enclosure. An aspiration is to study the pollution performance of bushings tainted by Nitrates.Arc flash tests of $1 \mathrm{kV}, 11 \mathrm{kV}, 17.5 \mathrm{kV}$ bushings are tainted by three types of salts such as $\mathrm{NaCl}, \mathrm{NaNO}_{3}, \mathrm{KNO}_{3}$. The morsels are negotiated under habitual environment as per IEC 60507. The impact of tainting salts with their solubility on Equivalent Salt Deposit Density (ESDD) and bushings arc flash voltage are scrutinized. The effect of tainted salts on arc flash fruition, the sway of volume conductivity and Equivalent Salt Deposit Density (ESDD) under different percentages are also scrutinized. The research upshot reveals that the Equivalent Salt Deposit Density (ESDD) rate escalated with escalating salt content. When salt concentration gets escalated then conductivity also get escalated. When Equivalent Salt Deposit Density (ESDD) get Escalated then the arc flash voltage and leakage current get slacken. Finally, the graphs are drawn between ESDD and Arc flash voltage, Conductivity and Salt concentration, Arc flash voltage and Leakage current are obtained using MATLAB software.
\end{abstract}

Keywords:Bushing, Tainted, Pollution arc flash, ESDD.

\section{INTRODUCTION}

In wattage, a bushing is comrade in nursing caulking device that enables comrade in nursing electrical conductor to pass safely through a grounded conducting barrier like the case of an electrical device or circuit breaker. Bushings area unit usually made of porcelain[1]; although alternative caulking materials are attainable, typically ceramic ware is employed. A bushing should be designed to resist the electrical intensity made within the caulking. Once any earthed material is gift, because the strength of the electrical field will increase, escape methods could develop inside the insulation. If the energy of the escape path overcomes the caulking strength of the insulation, it's going to puncture the insulation and permit the voltage to conduct to the closest earthed material inflicting burning and arcing.

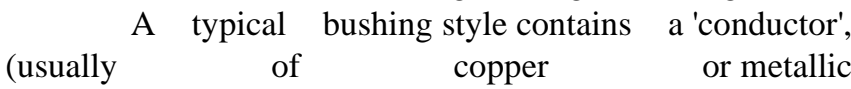
element, sometimes of different conductive materials), encir cled by insulation, apart from the terminal ends. Caulked bushings are often put in either indoor, or outdoor, and also

Revised Manuscript Received on December 30, 2019.

* Correspondence Author

Nikkitha.R*, High Voltage Engineering, National Engineering College, Kovilpatti,India.

Dr.L.Kalaivani, EEE, National Engineering College, Kovilpatti, India.

(C) The Authors. Published by Blue Eyes Intelligence Engineering and Sciences Publication (BEIESP). This is an open access article under the CC BY-NC-ND license (http://creativecommons.org/licenses/by-nc-nd/4.0/) the choice of caulking is going to be determined by the locale of the installation and also the electrical service duty on the bushing.

Ceramic ware was originally used because of its properties of being runproof to wetness [4]once sealed by laid-off glaze, and low producing price. A basic ceramic ware bushing may be a hollow ceramic ware form that matches through a hole during a wall or metal case, permitting a conductor to pass through its center, and connect at each end to different instrumentation. Bushings of this sort area unit usually product of wet-process laid-off ceramic ware, that is then glazed. A semi-conducting glaze could also be accustomed assist in equalizing the electrical potential gradient on the length of the bushing. Once pollution is wetted by rain[5] or fog, there's an unsought flow of current over the surface of the insulation of bushing is named run current. A discharge happens between the live finish of the bushing and another finish of the bushing that cause slacken of Arc flash voltage within the bushing.

\section{MORELS, TENTATIVE SETUP AND PROCEDURE}

\section{A. Morels}

The morels taken were three porcelain type of bushings such as $1 \mathrm{kV}, 11 \mathrm{kV}$ and $17.5 \mathrm{kV}$ respectively.

\section{B. Tentative Setup}

An ac artificial pollution[6], [7]feat takes a look at with non-uniform pollution distribution was administrated in bushings.The power supply is given as per Fig. 1. Fig. 1 shows the tentative setuphaving coupling capacitance, high voltage test transformer, and set up to hold a bushing. The transformer rated voltage is $2 \times 0.22 / 100 / 0.22 \mathrm{kV}$, rated current $2 \times 22.8 / 0.1 \mathrm{~A}$, rated output is $10 \mathrm{kVA}$. A testing transformer is employed to provide AC, DC, and impulse voltage. Supply is given to bushing by suggests that of the transformer. The management table is employed to regulate and operate high voltage AC equipment. The test setup is controlled by means of management table. The Arc flash voltage and leakage current may be noted from the management table.

\section{Test Procedure}

The transformer yieldextreme is connected to the top notch of the bushing. Before the test, all the test morels were cleaned and desiccatedspontaneously. The bushings were contaminated nonuniformly by suggests thatof conditional brushing methodology. 


\section{Influence of Arc Flash Performance and ESDD Measurement of Bushings Tainted by Nitrates}

The required quantity of $\mathrm{NaCl}$ or Nitrates and china stone were reckonedconsistence with the ratios by means that of electronic weight balancer. $\mathrm{NaCl}$ or Nitrates and china stone mixed with neededpure water and coated on the facial of the bushing by brushing methodology. When twenty-four hours of natural drying, the bushing is subjected to pollution performance check. The bushing used for testing is dangledupright on

the test setup and wetted by pure water. The high voltage is enforced slowly from zero voltage tillwhole Arc flash stumbles on the bushing surface. At Arc flash, the voltage is filed.The technique used for pollution could be a solid layer method[8], [9]. Brushing technique is finished, before beginning of pollution check, the load of $\mathrm{NaCl}$ (Sodium Chloride) and china stone is weighedby suggests that of weighing balance.The brush used for coating should be washed and cleaned with the pure water and artefact.

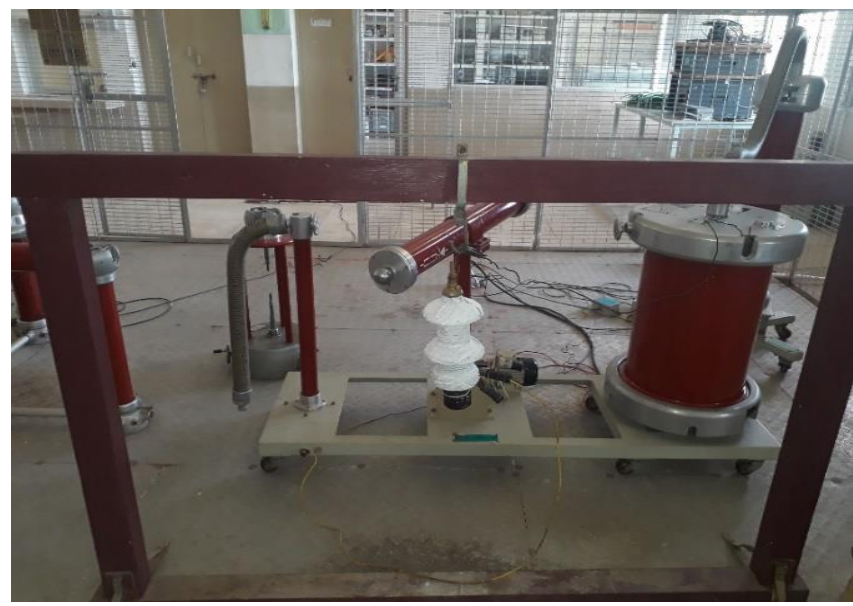

Fig. 1. Tentative setup for pollution testing

\section{EVALUATED PARAMETERS}

\section{A. Conductivity Measurement}

Conductivity of associate degree solution resolution could be a live of its endowment to charge electricity. The SI unit of conduction is Siemens per meter $(\mathrm{S} / \mathrm{m})$.Conductivity measurements are used most often in several industrial aura operation as a quick, cheap and reliable manner of mensuration the ionic during a solution.The electrical conduction of a solution of an electrolyte is measured by determinant the resistance of the answer between 2 flat or cylindrical electrodes separated by a hard and fast distance. Associate alternating voltage is hired so as to avoid electrolysis.The resistance is deliberated by a conductivity meter where Equation (2) shows the volume conductivity at $20^{\circ} \mathrm{C}$.

The specific conductance (conductivity), $\kappa$ (kappa) is the reciprocal of the specific resistance are shown in Equation (1).

$$
\begin{aligned}
& \mathrm{K}=\frac{1}{\mathrm{p}}=\frac{C}{R}(1) \\
& \sigma_{20^{\circ} \mathrm{C}}=\sigma_{\theta^{\circ} \mathrm{C}}[1-\mathrm{b}(\theta-20)](2)
\end{aligned}
$$

$\theta$ is the solution temperature $(\mathrm{C}), \sigma \theta$ is the volume conductivity [2], [3] at the temperature of $\theta^{\circ} \mathrm{C}(\mathrm{S} / \mathrm{m}), \sigma 20$ is the volume conductivity at the temperature of $20^{\circ} \mathrm{C}(\mathrm{S} / \mathrm{m})$.

\section{B. ESDD Measurement}

Pollution degree is determined by an equivalent salt deposit density which is detached from the insulating shoal of the bushing. After Arc flash examining, the contaminated bushings were desiccated under dazzling sunlight and the torrid granules of salts and china stone are assembled from the elite and basic surface of the bushing independently was assembled by brushing them off with a miniature paintbrush. The assembled salt deposits were then softening in $40 \mathrm{ml}$ of pure water. The conductivity measuring instrument was used to quota the conductivity of each assembled salt solutionwhere Equation (3) shows ESDD value [10].

\section{ESDD $=\left(\right.$ Sa $\times$ Volincm $\left.^{3}\right) /$ Areaincm $^{2}(3)$}

Vol - is the volume of the solution in $\mathrm{cm}^{3}$,

Area - is the area of the cleaned surface in $\mathrm{cm}^{2}$,

Sa - Salinity of the solution,

$$
\begin{aligned}
& S a=\left(5.7 \times \sigma_{20^{\circ} \mathrm{C}}\right)^{1.03}(4) \\
& \mathrm{b}=-3.200 \times 10^{-8}(\theta)^{3}+1.032 \times 10^{-5}(\theta)^{2}+-8.272 \times 10^{-4}(\theta) \\
& +3.544 \times 10^{-2}(5)
\end{aligned}
$$

where Equation (5) shows the factor depending on the temperature of $\theta$ and Equation (4) shows the salinity of the solution.

\section{Voltage at Standard Room Temperature and Pressure}

Classic action for temperature and pressure are classic sets of action for empirical measurements to be authorize to permit comparisons to be created betwixt different sets of instruction. The foremost used standards are those of the International Union of Pure and Applied Chemistry (IUPAC) and also the National Institute of Standards and Technology (NIST), although these are not universally accepted standards. Hence the Equation (6) and (7) shows the Voltage at standard temperature and pressure and Equation (7) shows the air density correction factor.

$\mathrm{V}_{\mathrm{STP}}=\mathrm{V}_{\mathrm{RTP}} *(\mathrm{HCF} / \mathrm{ADCF})(6)$

$\mathrm{ADCF}=(0.293 * 760) /(273+\mathrm{T})(7)$

$\mathrm{V}_{\text {sTP }}=$ Voltage at standard temperature pressure

$\mathrm{V}_{\mathrm{RTP}}=$ Voltage at Room temperature pressure

HCF $=1$, Humidity correction factor

$\mathrm{ADCF}=$ Air density correction factor

$\mathrm{T}=$ Room Temperature

where, 


\section{RESULTS AND DISCUSSION}

\section{A. Arc Flash Test on Sodium Chloride}

$\mathrm{NaCl}$ salt is taken with different combinations like $5 \mathrm{~g}, 10 \mathrm{~g}$, $15 \mathrm{~g}, 20 \mathrm{~g}, 30 \mathrm{~g}$ with Kaolin at constant $10 \mathrm{~g}$. Then the mixture is mixed with $15 \mathrm{ml}$ distilled water. The mixture is coated on the bushings using brushing method[10], [11] and then dried for 24hrs.After 24hrs drying Arc flash voltage and Leakage current is obtained by testing the test objects.Table. I - V shows $5,10,15,20,30 \mathrm{~g} \mathrm{NaCl}$ with

10g Kaolin.Depending upon the temperature variations dry and wet conditions changes simultaneously with respect to atmospheric condition. So, the Arc flash voltage at room temperature also get changed. Hence the Arc flash voltage is converted into standard temperature and pressure and it is listed in table, table. VI - X shows Arc flash voltage at standard room temperature and pressure for 5,10,15,20,30g $\mathrm{NaCl}$.

\section{B.Arc Flash Test on Potassium Nitrate}

$\mathrm{KNO}_{3}$ salt is taken with different combinations like $5 \mathrm{~g}$, $10 \mathrm{~g}, 15 \mathrm{~g}, 20 \mathrm{~g}, 30 \mathrm{~g}$ with Kaolin at constant $10 \mathrm{~g}$. Then the mixture is mixed with $15 \mathrm{ml}$ distilled water. The mixture is coated on the bushings using brushing method and then dried for 24hrs. After 24hrs drying Arc flash voltage and Leakage current is obtained by testing the test objects. Table. XI - XV shows 5,10,15,20,30g KNO3 with 10g Kaolin.

Depending upon the temperature variations dry and wet conditions changes simultaneously with respect to atmospheric condition. So, the Arc flash voltage at room temperature also get changed. Hence the Arc flash voltage is converted into standard temperature and pressure and it is shown in table, table. XVI - XX shows Arc flash voltage at standard room temperature and pressure for 5,10,15,20,30g $\mathrm{KNO}_{3}$.

\section{Arc Flash Test on Sodium Nitrate}

$\mathrm{NaNO}_{3}$ salt is taken with different combinations like $5 \mathrm{~g}$, $10 \mathrm{~g}, 15 \mathrm{~g}, 20 \mathrm{~g}$, 30g with Kaolin at constant $10 \mathrm{~g}$.Then the mixture is mixed with $15 \mathrm{ml}$ distilled water. The mixture is coated on the bushings using brushing method and then dried for 24hrs.After 24hrs drying Arc flash voltage and Leakage current is obtained by testing the test objects. Table. XXI XXV shows 5,10,15,20,30g $\mathrm{NaNO}_{3}$ with $10 \mathrm{~g}$ Kaolin.

Depending upon the temperature variations dry and wet conditions changes simultaneously with respect to atmospheric condition. So, the Arc flash voltage at room temperature also get changed. Hence the Arc flash voltage is converted into standard temperature and pressure and it is listed in table, table. XVI - XXX shows Arc flash voltage at standard room temperature and pressure for 5,10,15,20,30g $\mathrm{KNO}_{3}$.

Table- I: $5 \mathrm{~g} \mathrm{NaCl}$ with $10 \mathrm{~g}$ Kaolin at $31^{\circ} \mathrm{C}$

\begin{tabular}{|c|c|c|c|c|c|c|}
\hline $\begin{array}{c}\text { Rating of } \\
\text { bushing in } \\
\mathbf{k V}\end{array}$ & $\begin{array}{c}\text { Primary } \\
\text { voltage } \\
\mathbf{( k V )}\end{array}$ & $\begin{array}{c}\text { Arc Flash } \\
\text { voltage } \\
\mathbf{( k V )}\end{array}$ & $\begin{array}{c}\text { Leakage } \\
\text { current } \\
\mathbf{( m A )}\end{array}$ & $\begin{array}{c}\text { Weight } \\
\mathbf{( g )}\end{array}$ & $\begin{array}{c}\text { Conductivity } \\
(\boldsymbol{\mu s} / \mathbf{c m})\end{array}$ & $\begin{array}{c}\text { ESDD } \\
(\mathbf{m g} / \mathbf{c m} \wedge \mathbf{2})\end{array}$ \\
\hline 1 & 56 & 28.5 & 15.3 & 0.6 & 0.856 & 0.01486 \\
\hline 11 & 193 & 91.5 & 48 & 0.2 & 0.342 & 0.00577 \\
\hline 17.5 & 217 & 110 & 60 & 0.1 & 0.215 & 0.00204 \\
\hline
\end{tabular}

Table- II: $10 \mathrm{~g} \mathrm{NaCl}$ with $10 \mathrm{~g}$ Kaolin at $36^{\circ} \mathrm{C}$

\begin{tabular}{|c|c|c|c|c|c|c|}
\hline $\begin{array}{c}\text { Rating of } \\
\text { bushing in } \\
\mathbf{k V}\end{array}$ & $\begin{array}{c}\text { Primary } \\
\text { voltage } \\
\mathbf{( k V )}\end{array}$ & $\begin{array}{c}\text { Arc Flash } \\
\text { voltage } \\
\mathbf{( k V )}\end{array}$ & $\begin{array}{c}\text { Leakage } \\
\text { current } \\
\mathbf{( m A )}\end{array}$ & $\begin{array}{c}\text { Weight } \\
\mathbf{( g )}\end{array}$ & $\begin{array}{c}\text { Conductivity } \\
(\boldsymbol{\mu} \mathbf{s} / \mathbf{c m})\end{array}$ & $\begin{array}{c}\text { ESDD } \\
(\mathbf{m g} / \mathbf{c m} \wedge \mathbf{2})\end{array}$ \\
\hline 1 & 50 & 23.5 & 11.5 & 1 & 1.473 & 0.026 \\
\hline 11 & 156 & 73 & 35.5 & 0.3 & 0.428 & 0.0072 \\
\hline 17.5 & 180.5 & 84.5 & 43.5 & 0.2 & 0.457 & 0.0077 \\
\hline
\end{tabular}

Table- III: $15 \mathrm{~g} \mathrm{NaCl}$ with $10 \mathrm{~g}$ Kaolin at $44^{\circ} \mathrm{C}$

\begin{tabular}{|c|c|c|c|c|c|c|}
\hline $\begin{array}{c}\text { Rating of } \\
\text { bushing in } \\
\mathbf{k V}\end{array}$ & $\begin{array}{c}\text { Primary } \\
\text { voltage } \\
\mathbf{( k V )}\end{array}$ & $\begin{array}{c}\text { Arc Flash } \\
\text { voltage } \\
\mathbf{( k V )}\end{array}$ & $\begin{array}{c}\text { Leakage } \\
\text { current } \\
\mathbf{( m A )}\end{array}$ & $\begin{array}{c}\text { Weight } \\
\mathbf{( g )}\end{array}$ & $\begin{array}{c}\text { Conductivity } \\
(\boldsymbol{\mu s} / \mathbf{c m})\end{array}$ & $\begin{array}{c}\text { ESDD } \\
(\mathbf{m g} / \mathbf{c m} \wedge \mathbf{2})\end{array}$ \\
\hline 1 & 30 & 17 & 7.5 & 1.2 & 1.947 & 0.0353 \\
\hline 11 & 149.5 & 71.5 & 39.5 & 0.4 & 0.882 & 0.0153 \\
\hline 17.5 & 167.5 & 80.8 & 45 & 0.3 & 1.114 & 0.0195 \\
\hline
\end{tabular}


Influence of Arc Flash Performance and ESDD Measurement of Bushings Tainted by Nitrates

Table- IV: $20 \mathrm{~g} \mathrm{NaCl}$ with $10 \mathrm{~g}$ Kaolin at $36^{\circ} \mathrm{C}$

\begin{tabular}{|c|c|c|c|c|c|c|}
\hline $\begin{array}{c}\text { Rating of } \\
\text { bushing in } \\
\mathbf{k V}\end{array}$ & $\begin{array}{c}\text { Primary } \\
\text { voltage } \\
\mathbf{( k V )}\end{array}$ & $\begin{array}{c}\text { Arc Flash } \\
\mathbf{v o l t a g e} \\
\mathbf{( k V )}\end{array}$ & $\begin{array}{c}\text { Leakage } \\
\text { current } \\
\mathbf{( m A )}\end{array}$ & $\begin{array}{c}\text { Weight } \\
\mathbf{( g )}\end{array}$ & $\begin{array}{c}\text { Conductivity } \\
(\boldsymbol{\mu} \mathbf{s} / \mathbf{c m})\end{array}$ & $\begin{array}{c}\text { ESDD } \\
(\mathbf{m g} / \mathbf{c m} \wedge \mathbf{2})\end{array}$ \\
\hline 1 & 18.5 & 11 & 6.6 & 1.4 & 1.962 & 0.0349 \\
\hline 11 & 148 & 69.5 & 38.5 & 0.5 & 1.362 & 0.0233 \\
\hline 17.5 & 163.5 & 77.25 & 35.6 & 0.6 & 1.591 & 0.0281 \\
\hline
\end{tabular}

Table- V: $30 \mathrm{~g} \mathrm{NaCl}$ with $10 \mathrm{~g}$ Kaolin at $31^{\circ} \mathrm{C}$

\begin{tabular}{|c|c|c|c|c|c|c|}
\hline $\begin{array}{c}\text { Rating of } \\
\text { bushing in } \\
\mathbf{k V}\end{array}$ & $\begin{array}{c}\text { Primary } \\
\text { voltage } \\
\mathbf{( k V )}\end{array}$ & $\begin{array}{c}\text { Arc Flash } \\
\mathbf{v o l t a g e} \\
\mathbf{( k V )}\end{array}$ & $\begin{array}{c}\text { Leakage } \\
\text { current } \\
\mathbf{( m A )}\end{array}$ & $\begin{array}{c}\text { Weight } \\
\mathbf{( g )}\end{array}$ & $\begin{array}{c}\text { Conductivity } \\
(\boldsymbol{\mu s} / \mathbf{c m})\end{array}$ & $\begin{array}{c}\text { ESDD } \\
(\mathbf{m g} / \mathbf{c m} \wedge \mathbf{2})\end{array}$ \\
\hline 1 & 12.5 & 6.8 & 3.35 & 1.8 & 2.266 & 0.0405 \\
\hline 11 & 139.5 & 67 & 34.9 & 0.7 & 1.743 & 0.0309 \\
\hline 17.5 & 146.5 & 70.8 & 39.4 & 0.8 & 1.617 & 0.0286 \\
\hline
\end{tabular}

Table- VI: Arc flash voltage at standard room temperature and pressure for $5 \mathrm{~g} \mathrm{NaCl}$

\begin{tabular}{|c|c|c|}
\hline $\begin{array}{c}\text { Rating of } \\
\text { Bushing in kV }\end{array}$ & Room temperature $^{\circ} \mathbf{C}$ & VsTP \\
\hline 1 & 31 & 38.9 \\
\hline 11 & 31 & 124.9 \\
\hline 17.5 & 31 & 150.2 \\
\hline
\end{tabular}

Table- VII: Arc flash voltage at standard room temperature and pressure for $10 \mathrm{~g} \mathrm{NaCl}$

\begin{tabular}{|c|c|c|}
\hline $\begin{array}{c}\text { Rating of } \\
\text { Bushing in kV }\end{array}$ & Room temperature $^{\circ} \mathbf{C}$ & VsTP \\
\hline 1 & 36 & 32.61 \\
\hline 11 & 36 & 101.3 \\
\hline 17.5 & 36 & 117.3 \\
\hline
\end{tabular}

Table- VIII: Arc flash voltage at standard room temperature and pressure for $15 \mathrm{~g} \mathrm{NaCl}$

\begin{tabular}{|c|c|c|}
\hline $\begin{array}{c}\text { Rating of } \\
\text { Bushing in kV }\end{array}$ & Room temperature $^{\circ} \mathbf{C}$ & V STP \\
\hline 1 & 44 & 24.2 \\
\hline 11 & 44 & 101 \\
\hline 17.5 & 44 & 115.1 \\
\hline
\end{tabular}

Table- IX: Arc flash voltage at standard room temperature and pressure for $20 \mathrm{~g} \mathrm{NaCl}$

\begin{tabular}{|c|c|c|}
\hline $\begin{array}{c}\text { Rating of } \\
\text { Bushing in kV }\end{array}$ & Room temperature $^{\circ} \mathbf{C}$ & V $_{\text {STP }}$ \\
\hline 1 & 36 & 15.3 \\
\hline 11 & 36 & 92.6 \\
\hline 17.5 & 36 & 107.2 \\
\hline
\end{tabular}

Table- X: Arc flash voltage at standard room temperature and pressure for $30 \mathrm{~g} \mathrm{NaCl}$

\begin{tabular}{|c|c|c|}
\hline $\begin{array}{c}\text { Rating of } \\
\text { Bushing in kV }\end{array}$ & Room temperature $^{\circ} \mathbf{C}$ & V sTP \\
\hline 1 & 31 & 9.3 \\
\hline 11 & 31 & 91.5 \\
\hline 17.5 & 31 & 96.7 \\
\hline
\end{tabular}




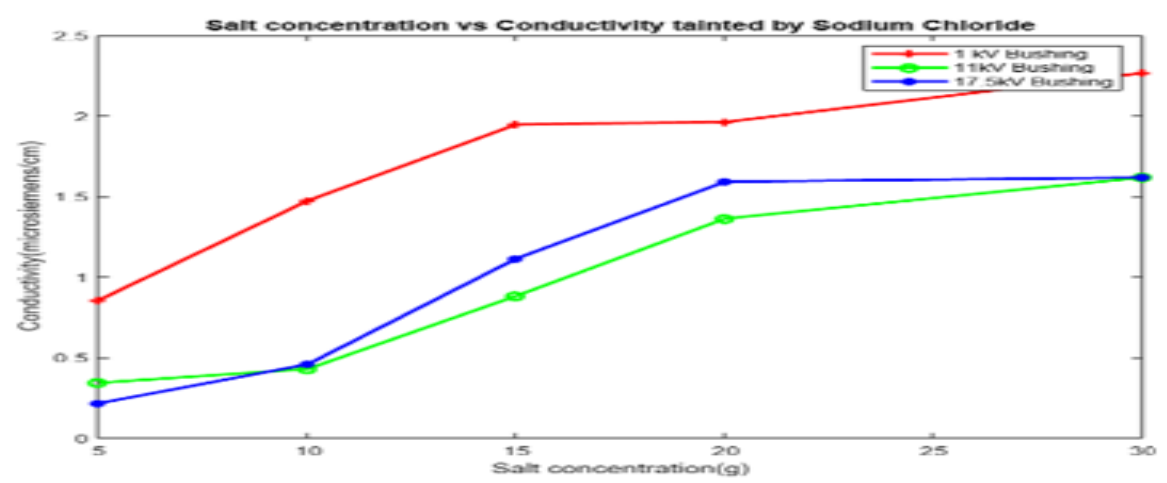

Fig. 2. Salt concentration and conductivity tainted by sodium chloride

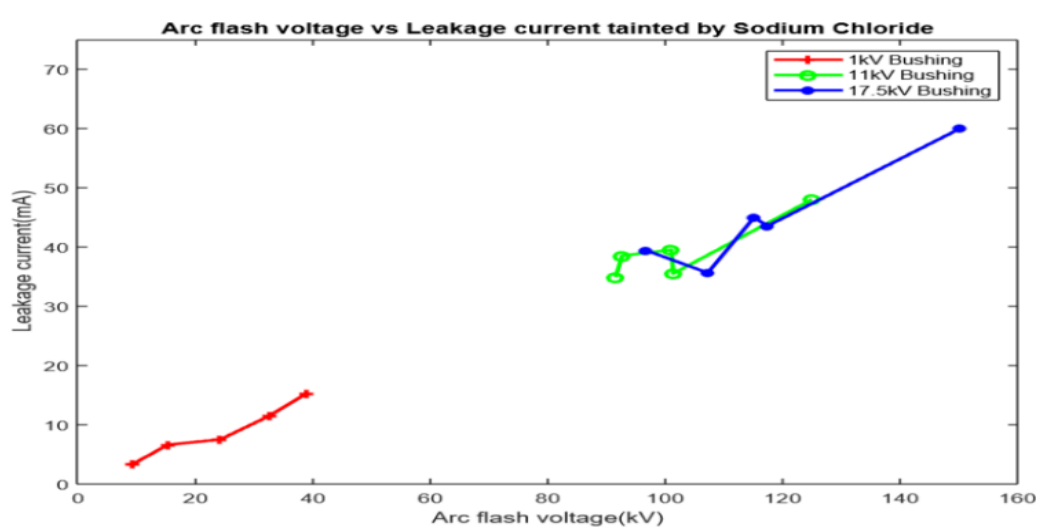

Figure. 3. Arc flash voltage and leakage current tainted by sodium chloride

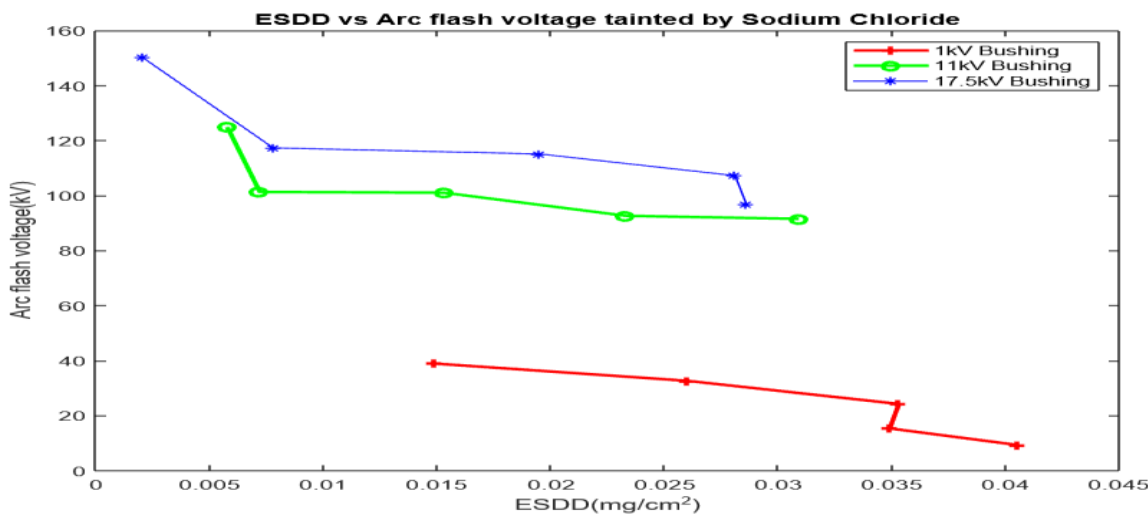

Fig. 4. ESDD and arc flash voltage tainted by sodium chloride

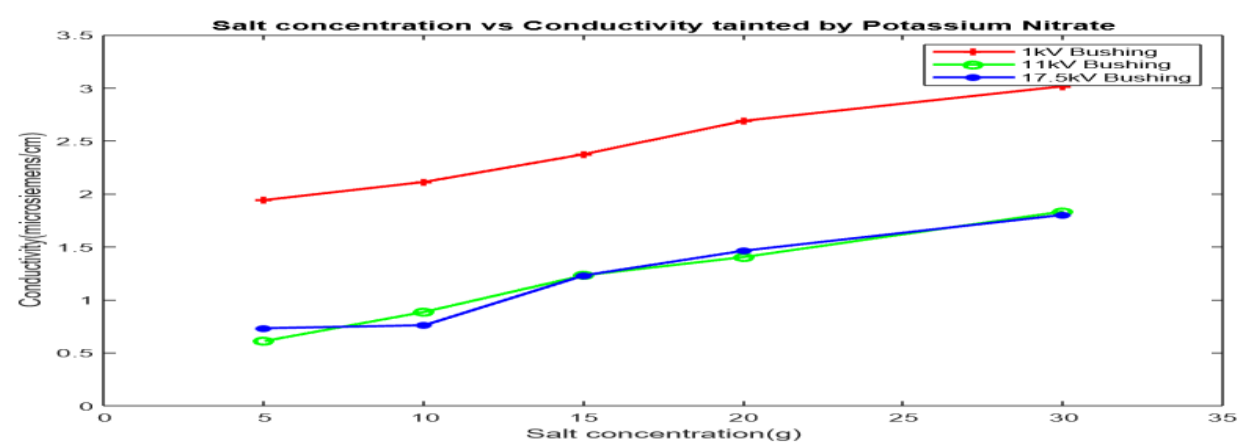

Fig. 5. Salt concentration and conductivity tainted by potassium nitrate 
Influence of Arc Flash Performance and ESDD Measurement of Bushings Tainted by Nitrates

Table- XI: $5 \mathrm{~g} \mathrm{KNO3}$ with $10 \mathrm{~g}$ Kaolin at $32^{\circ} \mathrm{C}$

\begin{tabular}{|c|c|c|c|c|c|c|}
\hline $\begin{array}{c}\text { Rating of } \\
\text { bushing in } \\
\mathbf{k V}\end{array}$ & $\begin{array}{c}\text { Primary } \\
\text { voltage } \\
\mathbf{( k V )}\end{array}$ & $\begin{array}{c}\text { Arc Flash } \\
\text { voltage } \\
\mathbf{( k V )}\end{array}$ & $\begin{array}{c}\text { Leakage } \\
\text { current } \\
\mathbf{( m A )}\end{array}$ & $\begin{array}{c}\text { Weight } \\
\mathbf{( g )}\end{array}$ & $\begin{array}{c}\text { Conductivity } \\
(\mathbf{\mu s} / \mathbf{c m})\end{array}$ & $\begin{array}{c}\text { ESDD } \\
(\mathbf{m g} / \mathbf{c m} \wedge \mathbf{2})\end{array}$ \\
\hline 1 & 23 & 14.5 & 9.35 & 0.2 & 1.941 & 0.0345 \\
\hline 11 & 151.5 & 71.5 & 43.05 & 0.1 & 0.610 & 0.01048 \\
\hline 17.5 & 191.5 & 91.5 & 55 & 0.1 & 0.731 & 0.01263 \\
\hline
\end{tabular}

Table- XII: $10 \mathrm{~g} \mathrm{KNO} 3$ with $10 \mathrm{~g}$ Kaolin at $32^{\circ} \mathrm{C}$

\begin{tabular}{|c|c|c|c|c|c|c|}
\hline $\begin{array}{c}\text { Rating of } \\
\text { bushing in } \\
\mathbf{k V}\end{array}$ & $\begin{array}{c}\text { Primary } \\
\text { voltage } \\
\mathbf{( k V )}\end{array}$ & $\begin{array}{c}\text { Arc flash } \\
\text { voltage } \\
\mathbf{( k V )}\end{array}$ & $\begin{array}{c}\text { Leakage } \\
\text { current } \\
\mathbf{( m A )}\end{array}$ & $\begin{array}{c}\text { Weight } \\
\mathbf{( g )}\end{array}$ & $\begin{array}{c}\text { Conductivity } \\
(\boldsymbol{\mu} \mathbf{s} / \mathbf{c m})\end{array}$ & $\begin{array}{c}\text { ESDD } \\
(\mathbf{m g} / \mathbf{c m} \wedge \mathbf{2})\end{array}$ \\
\hline 1 & 22.5 & 11.5 & 6.25 & 0.7 & 2.112 & 0.0376 \\
\hline 11 & 149.5 & 69 & 40.9 & 0.1 & 0.885 & 0.01538 \\
\hline 17.5 & 161 & 76 & 40.1 & 0.1 & 0.759 & 0.01313 \\
\hline
\end{tabular}

Table- XIII: $15 \mathrm{~g} \mathrm{KNO} 3$ with $10 \mathrm{~g}$ Kaolin at $34^{\circ} \mathrm{C}$

\begin{tabular}{|c|c|c|c|c|c|c|}
\hline $\begin{array}{c}\text { Rating of } \\
\text { bushing in } \\
\mathbf{k V}\end{array}$ & $\begin{array}{c}\text { Primary } \\
\text { voltage } \\
\mathbf{( k V )}\end{array}$ & $\begin{array}{c}\text { Arc flash } \\
\mathbf{v o l t a g e} \\
\mathbf{( k V )}\end{array}$ & $\begin{array}{c}\text { Leakage } \\
\text { current } \\
\mathbf{( m A )}\end{array}$ & $\begin{array}{c}\text { Weight } \\
\mathbf{( g )}\end{array}$ & $\begin{array}{c}\text { Conductivity } \\
(\boldsymbol{\mu s} / \mathbf{c m})\end{array}$ & $\begin{array}{c}\text { ESDD } \\
(\mathbf{m g} / \mathbf{c m} \wedge \mathbf{2})\end{array}$ \\
\hline 1 & 11 & 6.9 & 4.7 & 1.7 & 2.374 & 0.0425 \\
\hline 11 & 118.5 & 61.5 & 35 & 0.2 & 1.231 & 0.02161 \\
\hline 17.5 & 134.5 & 65.5 & 34.3 & 0.2 & 1.230 & 0.0215 \\
\hline
\end{tabular}

Table- XIV: $20 \mathrm{~g} \mathrm{KNO}_{3}$ with $10 \mathrm{~g}$ Kaolin at $33^{\circ} \mathrm{C}$

\begin{tabular}{|c|c|c|c|c|c|c|}
\hline $\begin{array}{c}\text { Rating of } \\
\text { bushing in } \\
\mathbf{k V}\end{array}$ & $\begin{array}{c}\text { Primary } \\
\text { voltage } \\
\mathbf{( k V )}\end{array}$ & $\begin{array}{c}\text { Arc flash } \\
\text { voltage } \\
\mathbf{( k V )}\end{array}$ & $\begin{array}{c}\text { Leakage } \\
\text { current } \\
\mathbf{( m A )}\end{array}$ & $\begin{array}{c}\text { Weight } \\
\mathbf{( g )}\end{array}$ & $\begin{array}{c}\text { Conductivity } \\
(\boldsymbol{\mu s} / \mathbf{c m})\end{array}$ & $\begin{array}{c}\text { ESDD } \\
(\mathbf{m g} / \mathbf{c m} \wedge \mathbf{2})\end{array}$ \\
\hline 1 & 12 & 7 & 3.75 & 2.7 & 2.689 & 0.0483 \\
\hline 11 & 130 & 61 & 34.1 & 0.3 & 1.403 & 0.02473 \\
\hline 17.5 & 135 & 63.5 & 33.5 & 0.3 & 1.463 & 0.0258 \\
\hline
\end{tabular}

Table- XV: $30 \mathrm{~g} \mathrm{KNO} 3$ with $10 \mathrm{~g}$ Kaolin at $30^{\circ} \mathrm{C}$

\begin{tabular}{|c|c|c|c|c|c|c|}
\hline $\begin{array}{c}\text { Rating of } \\
\text { bushing in } \\
\mathbf{k V}\end{array}$ & $\begin{array}{c}\text { Primary } \\
\text { voltage } \\
\mathbf{( k V )}\end{array}$ & $\begin{array}{c}\text { Arc flash } \\
\text { voltage } \\
\mathbf{( k V )}\end{array}$ & $\begin{array}{c}\text { Leakage } \\
\text { current } \\
\mathbf{( m A )}\end{array}$ & $\begin{array}{c}\text { Weight } \\
\mathbf{( g )}\end{array}$ & $\begin{array}{c}\text { Conductivity } \\
(\boldsymbol{\mu s} / \mathbf{c m})\end{array}$ & $\begin{array}{c}\text { ESDD } \\
(\mathbf{m g} / \mathbf{c m} \wedge \mathbf{2})\end{array}$ \\
\hline 1 & 8 & 3.85 & 0.25 & 3.3 & 3.016 & 0.054 \\
\hline 11 & 98 & 50.532 & 33.65 & 0.8 & 1.834 & 0.0325 \\
\hline 17.5 & 122.5 & 58.532 & 32 & 0.7 & 1.802 & 0.0320 \\
\hline
\end{tabular}

Table- XVI: Arc flash voltage at standard room temperature and pressure for $5 \mathrm{~g}$ KNO3

\begin{tabular}{|c|c|c|}
\hline $\begin{array}{c}\text { Rating of } \\
\text { Bushing in kV }\end{array}$ & Room temperature $^{\circ} \mathbf{C}$ & V $_{\text {STP }}$ \\
\hline $\mathbf{1}$ & 32 & 19.860 \\
\hline $\mathbf{1 1}$ & 32 & 97.93 \\
\hline $\mathbf{1 7 . 5}$ & 32 & 125.32 \\
\hline
\end{tabular}

Table- XVII: Arc flash voltage at standard room temperature and pressure for $10 \mathrm{~g} \mathrm{KNO}_{3}$

\begin{tabular}{|c|c|c|}
\hline $\begin{array}{c}\text { Rating of } \\
\text { Bushing in kV }\end{array}$ & Room temperature $^{\circ} \mathbf{C}$ & VsTP \\
\hline $\mathbf{1}$ & 32 & 15.751 \\
\hline $\mathbf{1 1}$ & 32 & 94.507 \\
\hline $\mathbf{1 7 . 5}$ & 32 & 104.09 \\
\hline
\end{tabular}


Table- XVIII: Arc flash voltage at standard room temperature and pressure for $15 \mathrm{~g} \mathrm{KNO}$

\begin{tabular}{|c|c|c|}
\hline $\begin{array}{l}\text { Rating of } \\
\text { Bushing in kV }\end{array}$ & Room temperature $^{\circ} \mathbf{C}$ & V STP \\
\hline $\mathbf{1}$ & 34 & 9.513 \\
\hline $\mathbf{1 1}$ & 34 & 85 \\
\hline $\mathbf{1 7 . 5}$ & 34 & 90.3 \\
\hline
\end{tabular}

Table- XIX: Arc flash voltage at standard room temperature and pressure for $20 \mathrm{~g} \mathrm{KNO}_{3}$

\begin{tabular}{|c|c|c|}
\hline $\begin{array}{c}\text { Rating of } \\
\text { Bushing in kV }\end{array}$ & Room temperature $^{\circ} \mathbf{C}$ & V $_{\text {sTP }}$ \\
\hline $\mathbf{1}$ & 33 & 9.619 \\
\hline $\mathbf{1 1}$ & 33 & 83.82 \\
\hline $\mathbf{1 7 . 5}$ & 33 & 87.259 \\
\hline
\end{tabular}

Table- XX: Arc flash voltage at standard room temperature and pressure for $30 \mathrm{~g} \mathrm{KNO}_{3}$

\begin{tabular}{|c|c|c|}
\hline $\begin{array}{c}\text { Rating of } \\
\text { Bushing in kV }\end{array}$ & Room temperature $^{\circ} \mathbf{C}$ & VsTP \\
\hline $\mathbf{1}$ & 30 & 5.2386 \\
\hline $\mathbf{1 1}$ & 30 & 68.715 \\
\hline $\mathbf{1 7 . 5}$ & 30 & 79.600 \\
\hline
\end{tabular}

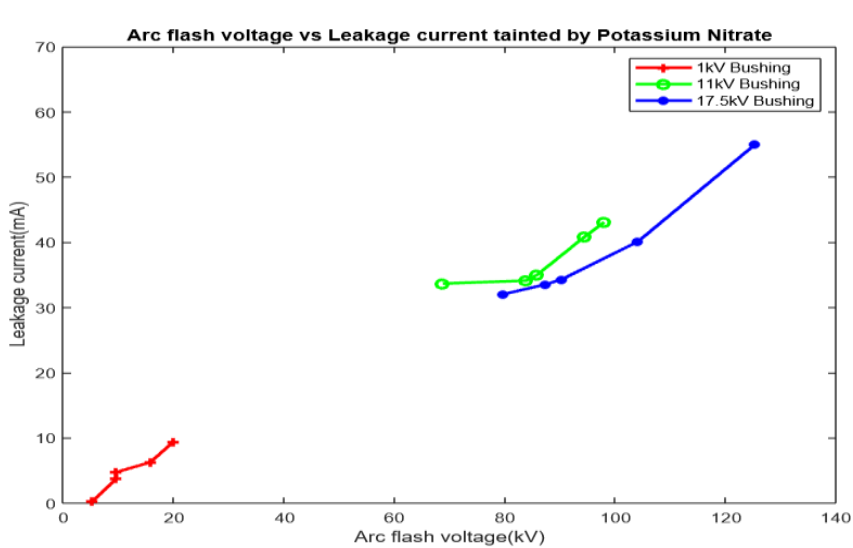

Fig. 6. Arc flash voltage and leakage current tainted by potassium nitrate

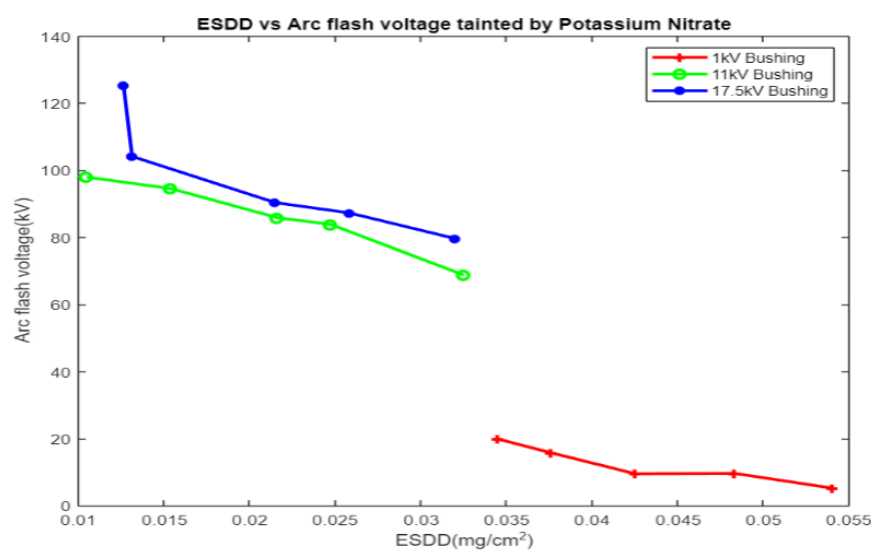

Fig. 7. ESDD and arc flash voltage tainted by potassium nitrate

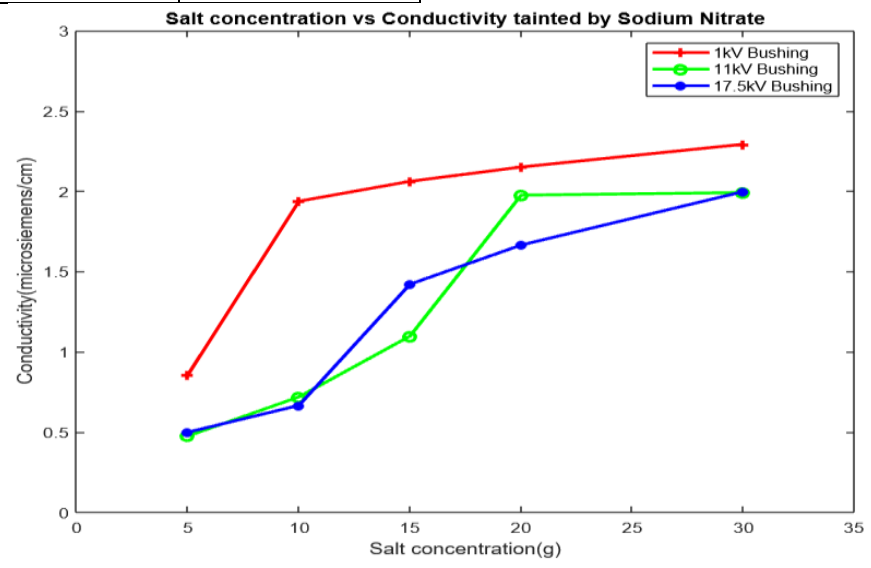

Fig. 8. Salt concentration and conductivity tainted by sodium nitrate 
Influence of Arc Flash Performance and ESDD Measurement of Bushings Tainted by Nitrates

Table- XXI: $5 \mathrm{~g} \mathrm{NaNO} 3$ with $10 \mathrm{~g}$ Kaolin at $33^{\circ} \mathrm{C}$

\begin{tabular}{|c|c|c|c|c|c|c|}
\hline $\begin{array}{c}\text { Rating of } \\
\text { bushing in } \\
\mathbf{k V}\end{array}$ & $\begin{array}{c}\text { Primary } \\
\text { voltage } \\
\mathbf{( k V )}\end{array}$ & $\begin{array}{c}\text { Arc flash } \\
\text { voltage } \\
\mathbf{( k V )}\end{array}$ & $\begin{array}{c}\text { Leakage } \\
\text { current } \\
\mathbf{( m A )}\end{array}$ & $\begin{array}{c}\text { Weight } \\
\mathbf{( g )}\end{array}$ & $\begin{array}{c}\text { Conductivity } \\
(\boldsymbol{\mu s} / \mathbf{c m})\end{array}$ & $\begin{array}{c}\text { ESDD } \\
(\mathbf{m g} / \mathbf{c m} \wedge \mathbf{2})\end{array}$ \\
\hline 1 & 10 & 6.25 & 2.8 & 0.4 & 0.854 & 0.01483 \\
\hline 11 & 150 & 72 & 44 & 0.1 & 0.474 & 0.00808 \\
\hline 17.5 & 186.5 & 88.5 & 50.5 & 0.2 & 0.498 & 0.008511 \\
\hline
\end{tabular}

Table- XXII: $10 \mathrm{~g} \mathrm{NaNO}_{3}$ with $10 \mathrm{~g}$ Kaolin at $35^{\circ} \mathrm{C}$

\begin{tabular}{|c|c|c|c|c|c|c|}
\hline $\begin{array}{c}\text { Rating of } \\
\text { bushing in } \\
\mathbf{k V}\end{array}$ & $\begin{array}{c}\text { Primary } \\
\text { voltage } \\
\mathbf{( k V )}\end{array}$ & $\begin{array}{c}\text { Arc flash } \\
\text { voltage } \\
\mathbf{( k V )}\end{array}$ & $\begin{array}{c}\text { Leakage } \\
\text { current } \\
\mathbf{( m A )}\end{array}$ & $\begin{array}{c}\text { Weight } \\
\mathbf{( g )}\end{array}$ & $\begin{array}{c}\text { Conductivity } \\
(\boldsymbol{\mu s} / \mathbf{c m})\end{array}$ & $\begin{array}{c}\text { ESDD } \\
(\mathbf{m g} / \mathbf{c m} \wedge \mathbf{2})\end{array}$ \\
\hline 1 & 10.5 & 5.5 & 2.9 & 0.8 & 1.937 & 0.03448 \\
\hline 11 & 171.5 & 80 & 49.8 & 0.1 & 0.718 & 0.0124 \\
\hline 17.5 & 175 & 82 & 50.9 & 0.1 & 0.655 & 0.0112 \\
\hline
\end{tabular}

Table- XXIII: $15 \mathrm{~g} \mathrm{NaNO} 3$ with $10 \mathrm{~g}$ Kaolin at $35^{\circ} \mathrm{C}$

\begin{tabular}{|c|c|c|c|c|c|c|}
\hline $\begin{array}{c}\text { Rating of } \\
\text { bushing in } \\
\mathbf{k V}\end{array}$ & $\begin{array}{c}\text { Primary } \\
\text { voltage } \\
\mathbf{( k V )}\end{array}$ & $\begin{array}{c}\text { Arc flash } \\
\text { voltage } \\
\mathbf{( k V )}\end{array}$ & $\begin{array}{c}\text { Leakage } \\
\text { current } \\
\mathbf{( m A )}\end{array}$ & $\begin{array}{c}\text { Weight } \\
\mathbf{( g )}\end{array}$ & $\begin{array}{c}\text { Conductivity } \\
(\boldsymbol{\mu s} / \mathbf{c m})\end{array}$ & $\begin{array}{c}\text { ESDD } \\
(\mathbf{m g} / \mathbf{c m} \wedge \mathbf{2})\end{array}$ \\
\hline 1 & 6.5 & 5.25 & 2.65 & 1.2 & 2.061 & 0.0367 \\
\hline 11 & 167.5 & 80 & 46.65 & 0.2 & 1.097 & 0.01919 \\
\hline 17.5 & 173 & 81.5 & 47.9 & 0.3 & 1.420 & 0.02504 \\
\hline
\end{tabular}

Table- XXIV: 20g NaNO 3 with $10 \mathrm{~g}$ Kaolin at $33^{\circ} \mathrm{C}$

\begin{tabular}{|c|c|c|c|c|c|c|}
\hline $\begin{array}{c}\text { Rating of } \\
\text { bushing in } \\
\mathbf{k V}\end{array}$ & $\begin{array}{c}\text { Primary } \\
\text { voltage } \\
\mathbf{( k V )}\end{array}$ & $\begin{array}{c}\text { Arc flash } \\
\text { voltage } \\
\mathbf{( k V )}\end{array}$ & $\begin{array}{c}\text { Leakage } \\
\text { current } \\
\mathbf{( m A )}\end{array}$ & $\begin{array}{c}\text { Weight } \\
\mathbf{( g )}\end{array}$ & $\begin{array}{c}\text { Conductivity } \\
\mathbf{( \boldsymbol { \mu } \mathbf { s } / \mathbf { c m } )}\end{array}$ & $\begin{array}{c}\text { ESDD } \\
(\mathbf{m g} / \mathbf{c m} \wedge \mathbf{2})\end{array}$ \\
\hline 1 & 10 & 6.45 & 2.8 & 1.4 & 2.150 & 0.03839 \\
\hline 11 & 168 & 79.5 & 47.15 & 0.3 & 1.975 & 0.03512 \\
\hline 17.5 & 185.5 & 89.5 & 53.3 & 0.2 & 1.664 & 0.02948 \\
\hline
\end{tabular}

Table- XXV: 30g NaNO 3 with $10 \mathrm{~g}$ Kaolin at $34^{\circ} \mathrm{C}$

\begin{tabular}{|c|c|c|c|c|c|c|}
\hline $\begin{array}{c}\text { Rating of } \\
\text { bushing in } \\
\mathbf{k V}\end{array}$ & $\begin{array}{c}\text { Primary } \\
\text { voltage } \\
\mathbf{( k V )}\end{array}$ & $\begin{array}{c}\text { Arc flash } \\
\text { voltage } \\
\mathbf{( k V )}\end{array}$ & $\begin{array}{c}\text { Leakage } \\
\text { current } \\
\mathbf{( m A )}\end{array}$ & $\begin{array}{c}\text { Weight } \\
\mathbf{( g )}\end{array}$ & $\begin{array}{c}\text { Conductivity } \\
(\boldsymbol{\mu s} / \mathbf{c m})\end{array}$ & $\begin{array}{c}\text { ESDD } \\
(\mathbf{m g} / \mathbf{c m} \wedge \mathbf{2})\end{array}$ \\
\hline 1 & 5.5 & 3.95 & 2.85 & 1.6 & 2.292 & 0.04100 \\
\hline 11 & 125.5 & 60.45 & 33 & 0.6 & 1.991 & 0.03547 \\
\hline 17.5 & 156.5 & 76 & 44.45 & 0.7 & 1.997 & 0.0355 \\
\hline
\end{tabular}

Table- XVI: Arc flash voltage at standard room temperature and pressure for $5 \mathrm{~g} \mathrm{NaNO}_{3}$

\begin{tabular}{|c|c|c|}
\hline Rating of Bushing in kV & Room temperature $^{\circ} \mathbf{C}$ & VsTP \\
\hline $\mathbf{1}$ & 33 & 8.588 \\
\hline $\mathbf{1 1}$ & 33 & 98.94 \\
\hline $\mathbf{1 7 . 5}$ & 33 & 121.613 \\
\hline
\end{tabular}

Table- XVII: Arc flash voltage at standard room temperature and pressure for $10 \mathrm{~g} \mathrm{NaNO}_{3}$

\begin{tabular}{|c|c|c|}
\hline Rating of Bushing in kV & Room temperature ${ }^{\circ} \mathbf{C}$ & VsTP \\
\hline $\mathbf{1}$ & 35 & 7.067 \\
\hline $\mathbf{1 1}$ & 35 & 110.652 \\
\hline $\mathbf{1 7 . 5}$ & 35 & 113.418 \\
\hline
\end{tabular}


Table- XVIII: Arc flash voltage at standard room temperature and pressure for $15 \mathrm{~g} \mathrm{NaNO}_{3}$

\begin{tabular}{|c|c|c|}
\hline $\begin{array}{c}\text { Rating of } \\
\text { Bushing in kV }\end{array}$ & $\begin{array}{c}\text { Room } \\
\text { temperature }^{\circ} \mathbf{C}\end{array}$ & VsTP \\
\hline $\mathbf{1}$ & 35 & 7.2615 \\
\hline $\mathbf{1 1}$ & 35 & 110.65 \\
\hline $\mathbf{1 7 . 5}$ & 35 & 112.72 \\
\hline
\end{tabular}

Table- XIX: Arc flash voltage at standard room temperature and pressure for $20 \mathrm{~g}$ NaNO3

\begin{tabular}{|c|c|c|}
\hline $\begin{array}{c}\text { Rating of } \\
\text { Bushing in kV }\end{array}$ & $\begin{array}{c}\text { Room } \\
\text { temperature }^{\circ} \mathbf{C}\end{array}$ & VsTP \\
\hline $\mathbf{1}$ & 33 & 8.863 \\
\hline $\mathbf{1 1}$ & 33 & 109.25 \\
\hline $\mathbf{1 7 . 5}$ & 33 & 122.98 \\
\hline
\end{tabular}

Table- XXX: Arc flash voltage at standard room temperature and pressure for $30 \mathrm{~g} \mathrm{NaNO}_{3}$

\begin{tabular}{|c|c|c|}
\hline $\begin{array}{c}\text { Rating of } \\
\text { Bushing in kV }\end{array}$ & $\begin{array}{c}\text { Room } \\
\text { temperature }^{\circ} \mathbf{C}\end{array}$ & VsTP \\
\hline $\mathbf{1}$ & 34 & 5.445 \\
\hline $\mathbf{1 1}$ & 34 & 83.33 \\
\hline $\mathbf{1 7 . 5}$ & 34 & 104.778 \\
\hline
\end{tabular}

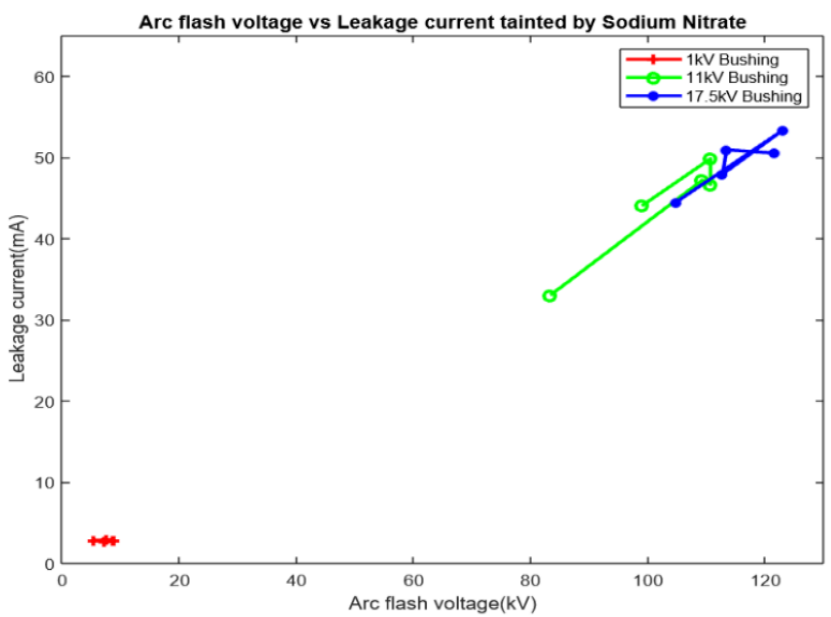

Fig. 9. Arc flash voltage and leakage current tainted by sodium nitrate

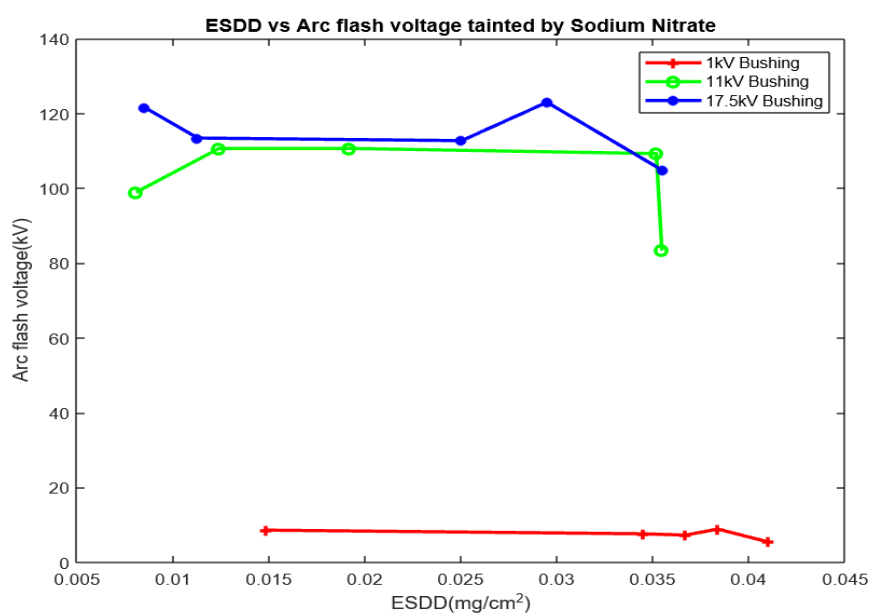

Fig. 10. ESDD and arc flash voltage tainted by sodium nitrate

From fig.2, 5 and 8 shows that the salt concentration in water determines its physical phenomenon. The larger the salt concentration, the upper the physical phenomenon. The physical phenomenon of an answer depends on the quantity of charge carriers (the concentrations of the ions), the quality of the charge carriers and their charge. In theory, conductivity ought to increase in direct proportion to concentration. Some ionic compounds aresoluble, which implies they dissolve in water. once these compounds dissolve, they dissociate, or break into their respective ions. As a category of compounds, salts are chemicals comprised of a metal and a nonmetal. The metal assumes a positive charge and is a cation whereas the nonmetal assumes a negative charge and is an anion.Fig. 3, 6 and 9 normally shows that when voltage get increases then current get decreases (minimum or zero), both are inversely proportional. At the same time, high voltage is passed on the pollution shoal of the bushing, then conducting channel is model on the shoal due to stress and it forms contamination layer due to the presence of salts. At this condition, leakage current flows on the shoal of the bushing and the amount of leak current flows on the shoal is expressed in terms of milliampere. So, flash over occurs on the shoal of bushing and the leak current get escalated.

When the applied voltage gets escalated then the leak current passes through the pollution shoal also get escalated and form a conducting channel.

From fig. 4, 7 and 10 shows that when ESDD values get increases then arc flash voltage gets decreases. When salt concentration get increases then the flashover occurs earlier.

Arc flash occurs quickly in $1 \mathrm{kV}$ bushing when compare to $11 \mathrm{kV}, 17.5 \mathrm{kV}$ bushing because it has shortest creepage distance hence the ESDD value get increases. Creepage distance is the shortest distance between two live conductors. $11 \mathrm{kV}$ and $17.5 \mathrm{kV}$ bushing has long creepage distance so the formation of conduction layer is discontinuing and the arc flash voltage get increases.

\section{CONCLUSION}

This work is positioned on the AC unrealcontamination test with non-uniform contamination on bushings, some conclusions are obtained as follows, 


\section{Influence of Arc Flash Performance and ESDD Measurement of Bushings Tainted by Nitrates}

- When salt concentration get increases then the conductivity also get increases drastically.

- The Equivalent Salt Deposit Density increases with increased salt concentration.

- The arc flash voltage decreases gradually when the Equivalent Salt Deposit Density rate increases.

- The arc flash voltage gets escalated and the leakage current also get escalated gradually.

- When comparing to clean bushings, the arc flash voltage for $1 \mathrm{kV}, 11 \mathrm{kV}, 17.5 \mathrm{kV}$ bushings decreases very rapidly when the bushings are tainted with $\mathrm{NaCl}, \mathrm{KNO}_{3}$ and $\mathrm{NaNO}_{3}$.

- From the above obtained results, the arc flash voltage for $\mathrm{KNO}_{3}>\mathrm{NaNO}_{3}>\mathrm{NaCl}$ respectively.

From this the conclusion can be made that the reaction of nitrates degrades the performance of bushings very quickly when comparing to $\mathrm{NaCl}$.

\section{REFERENCES}

1. Xingliang Jiang, Jihe Yuan, Lichun Shu, Zhijin Zhang, Jianlin Hu and Feng Mao, "Comparison of DC Pollution Flashover Performances of Various Types of Porcelain, Glass, and Composite Insulators", IEEE Transactions on Power Delivery, Vol.23, NO.2, pp. 1183-1190, 2008.

2. Zhijin Zhang, Dongdong Zhang and Xingliang Jiang, "Effects of Pollution Materials on the AC Flashover Performance of Suspension Insulators", IEEE Transactions on Dielectrics and Electrical Insulation, Vol.22, No.2, pp. 1000-1008, 2015.

3. Zhijin Zhang, XinhanQiao, Yi Zhang, Liang Tian, Dongdong Zhang and Xingliang Jiang, "AC flashover performance of different shed configurations of composite insulators under fan-shaped non-uniform pollution", IET Generation Transmission and Distribution, Vol.3, Iss.3, pp. 199-206, 2018.

4. W. Lampe, Senior Member, D. Wikstriim and B. Jacobson, "Field Distribution on an HVDC Wall Bushing During Laboratory Rain Tests",IEEE Transactions on Power Delivery, Vol.6, No.4, pp. 1531-1540, 1991.

5. H. M. Schneider A and E. Lux, "Mechanism of HVDC Wall Bushing Flashover in Nonuniform Rain", IEEE Transactions on Power Delivery, Vol.6, No.1, pp. 448-455, 1991.

6. Chris S. Engelbrecht and Ralf Hartings, "Pollution Tests for Coastal Conditions on an 800-kV Composite Bushing", IEEE Transactions on Power Delivery, Vol.18, NO.3, pp. 953-959, 2003.

7. Alok Ranjan Verma and Subba Reddy B, "Tracking and Erosion Resistance of LSR and HTV Silicone Rubber Samples under Acid Rain Conditions", IEEE Transactions on Dielectrics and Electrical Insulation, Vol.25, No.1, pp. 46-52, 2018.

8. S. Chandrasekar and C. Kalaivanan, "Partial Discharge Detection as a Tool to Infer Pollution Severity of Polymeric Insulators", IEEE Transactions on Dielectrics and Electrical Insulation, Vol.17, No.1, pp. 304-313, 2010.

9. D. Maadjoudj, A. Mekhaldi and M. Teguar, "Flashover Process and Leakage Current Characteristics of Insulator Model under Desert Pollution",IEEE Transactions on Dielectrics and Electrical Insulation, Vol.25, No.3, pp.1115-1123, 2018.

10. Dongdong Zhang, Zhijin Zhang, Xingliang Jiang, Zhongyi Yang and Youchao Liu, "Study on the Flashover Performance of Various Types of Insulators Polluted by Nitrates", IEEE Transactions on Dielectrics and Electrical Insulation, Vol.24, No.1, pp. 167-174, 2017.

11. Zhongyi Yang, XingliangJiang ,Xingbo Han , Zhijin Zhang and Jianlin $\mathrm{Hu}$, "Influence of pollution chemical components on AC Flashover performance of various types of insulators",IET Generation Transmission and Distribution, Vol.4, Iss.2, pp.105-112, 2019.

\section{AUTHORS PROFILE}

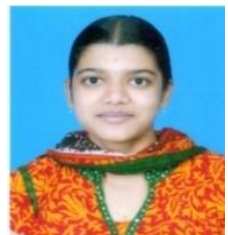

R.Nikkitha received BE Degree in electrical and electronics engineering in 2018 at National Engineering College, Kovilpatti. She is studying final year high voltage Engineering in National Engineering College, Kovilpatti. Her area of interest is Transformer.

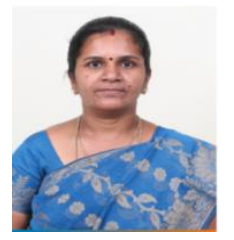

Dr.L.Kalaivanireceived her Ph.D. degree in Information andCommunication Engineering from Anna University, Chennai,Tamilnadu, India. She works as a Professor in Department of Electrical and Electronics Engineering, National Engineering College, kovilpatti, Tamilnadu, India. She is a member of various professional bodies and organized varied funded workshops and seminars. She acted as Co-Investigator and completed one funded project. She has wide publications in SCI, Scopus indexed Journals \& Conferences. She is an expert member in NBA and enhances the TLP. Currently, her research interests include Wireless networks and soft computing. 